\title{
Development of an aerodynamic control system for the evaluation of the Open Core Engineering technology
}

\author{
Prof. Dr.-Ing. Elmar Engels, M.Eng. Alexander-Nicolai Köhler \\ University of Applied Sciences Fulda, Leipziger Str. 123, D-36037, Germany
}

\begin{abstract}
This paper describes the development of an aerodynamic control system used for the evaluation and demonstration of the award winning Open Core Engineering (OCE) technology. The aerodynamic control system was designed with physical properties that allow using it in rapid-control-prototyping (RCP) environments as well as with industrial motion-control components. Open Core Engineering is a quite modern technology invented by Bosch Rexroth AG that supports software development on several platforms for industrial automation applications. A 3D printed alternative design is explained as well.
\end{abstract}

\section{Introduction and Objectives}

Based on the fact that information technology merges more and more with production and process automation technologies the Open Core Engineering (OCE) technology was developed supporting the ideas of Industry 4.0 within the area of industrial drives and controls. Even though OCE was developed for industrial applications it can be beneficially used in mechatronics research and development projects as well. In order to evaluate the features and usability of the OCE an aerodynamic test bench was developed that fits very well to use cases of industrial and scientific applications.

The use cases could be human machine interface (HMI) development, IEC 61131-3 software development, RCP with MATLAB ${ }^{\circledR}$ / Simulink $^{\circledR}$ or LabVIEW $^{\circledR}$, desktop software development like VBA for Microsoft Excel, Microsoft PowerPoint or Microsoft Access and remote control applications for mobile devices like iOS or Android based devices. From the perspective of mechatronic system developments these systems differ a lot in terms of programming paradigms, software development tools and languages as well as in performance classes. Performance class means that applications written in VBA for Excel or Java applications for smartphones cannot reach real-time properties as industrial motion-controllers - normally written in $\mathrm{C}, \mathrm{C}++$ or domain specific programming languages - can reach.

Thus the objective in this research project was to design a test bench that has a performance class not too restrictive for non-real-time applications and on the other hand being challenging enough to test the applicability of the OCE concept for higher performance industrial applications and to evaluate the usability. Fig. 1 shows a detail of the aerodynamic system in a horizontal orientation. The mechatronic requirements of its realization will be explain in the following sections.

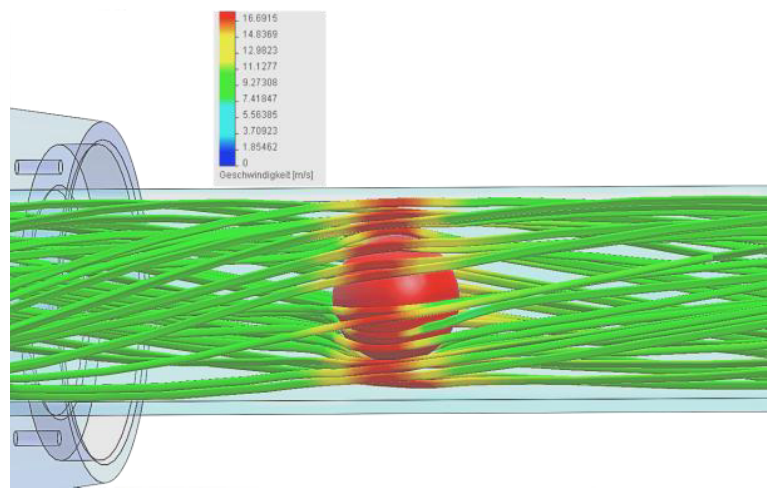

Figure 1: Aerodynamic control system Hoverball [1]

\section{Requirements and Design Approach}

There are a lot of mechatronic systems that could be considered to evaluate OCE with a test bench $[2,3]$. However as mentioned above it had to cover several software domains and it was required to use a control application with a good visualization of the process for demonstration purposes. The general requirements for the test bench were to use it as demonstrator for applications from the domains of industrial automation, control theory, electric drives and obviously for software engineering.

To break these requirements a bit more down the objectives were to evaluate it in the area of industrial automation with project configuration and commissioning, software development with IEC 61131-3 programming languages, HMI design and use of industrial sensors and actors. 
For the area of control theory it should be usable for typical tasks like modeling, analysis, simulation and identification of system parameters to mention only a few use cases. To perform these use cases the test bench should have some special characteristics like being instable or requiring control for stabilization, being nonlinear and having the possibility to apply system perturbation, disturbances and to apply a fix set-point control as well as a servo control.

Evaluation of usability for industrial drives means that typical functionality of drives should be usable. This does not automatically mean that an industrial drive has to be used for the aerodynamic system. It could mean that typical functions like position control, velocity control or synchronization modes could be used. Sophisticated functions like cams would be desirable as well.

Software development is an integral part of all the mentioned areas but does not mean that software development theories are the focus. This means as explained above that different kind of programming paradigms like functional design, object oriented design and model driven design should be usable with the test bench but this is more determined by the programming languages themselves than by the test bench. However, the test bench must be programmable with these languages. So the test bench should have properties that support these software languages.

\section{Motivation}

Starting point of the development of this mechatronic system was the idea of creating a test bench based on aerodynamic systems presented in $[4,5]$. The authors use micro-controller based electronics normally used for embedded system design. But they do not use components like industrial programmable-logiccontrollers (PLC) or industrial drives that is a key target in this project. The principle of an aerodynamic levitated ball has several advantages. First of all the system includes moving components that visualize the process immediately. The system can be operated at extra-lowvoltage and therefore in office environments. There are other test-benches like an inverted pendulum or a balanced ball that also visualize if control algorithms are applied properly. But a levitated ball is much slower in its reaction having at the same time a tendency to instability if the airflow is designed so.

Systems like magnetically levitated balls fall into the same class of application but they have the disadvantage that their movements are usually limited to a few millimeters meaning that the visible motion effect is pretty small compared to an aerodynamically levitated ball. Magnetically levitated balls do also have the disadvantage that the control cycle time has to be quite small within a range of typically $1 \mathrm{~ms}$ to $0.1 \mathrm{~ms}$. Particularly slow update frequencies of big jitter during the control process jeopardize the usability with non-realtime systems. Therefore the decision was made for an aerodynamic levitated ball with a dedicated construction set-up. The test bench was called Hoverball and has several properties that make it very well suited for the evaluation of the OCE technology.

\section{Realization of the Hoverball}

In the following section the mechanical construction and its components will be explained without going into details of the aerodynamic system. Fig. 2 shows a transparent view of the main mechanical components. Basically the structure is composed of the aluminum body and the transparent acryl pipe. The acryl pipe guides the air flow generated in the body. Within that air flow a ball can be hovered if the air flow is controlled.

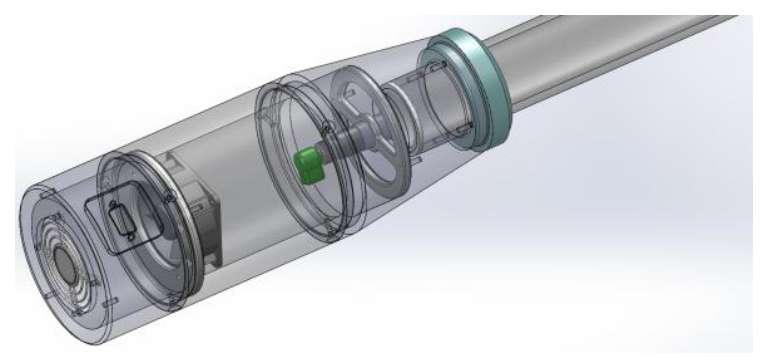

Figure 2: Mechanical concept of the Hoverball [1]

The body itself is split in the lower part, the center part and the top part. The lower part contains the electronic boards with power supply, signal conditioning and power electronics for a fan. Due to the position of the fan and the power electronics the fan has the side effect that it cools down the electronics.

The fan is hosted in the center part that has enough space to place additional components to regulate the air flow. Additional components could be a throttle flap to control the air flow or to make it even more turbulent or it could be a diffusor that would create a more laminar air flow. However the actual implementation was only used with and without diffusor. Obviously laminar air flow creates a more stable position of the ball and makes it slightly easier to find accurate controller parameters.

The upper part of the body contains the ultrasonic position sensor and a tightening mechanism for the acryl pipe. Normally it is difficult to place an ultrasonic positioning sensor in a small pipe as the radiation pattern of the sensor could detect edges of the construction or get interference of ultrasonic waves reflected at the cylindrical walls. The sensor used in this application has a radiation pattern that would exceed the diameter of the pipe by far but due to its position its beam enters the pipe without reflection and returns an acceptable signal quality for this application. The ultrasonic position sensor is programmable so the measurement range can be adjusted exactly to the length of the pipe and the sensor returns a $4 . .20 \mathrm{~mA}$ current signal proportional to the position of the ball.

As actuator to create the air flow a standard fan was used. It has the advantage that it keeps the noise level quite low and is pretty efficient versus a self-constructed motor with propeller. The fan speed can be modified due to a variation of the supply voltage. The operation voltage 
ranges from $6 . .24 \mathrm{~V}$ which covers the complete air flow range needed to control the ball position.

The fan supply voltage is generated by a classic linear power operational amplifier circuit. The power operational amplifier has a high bandwidth and does not create any critical system dynamics.

The final important mechanical component is the ball itself that has to be levitated by the air flow. The ball is a standard but painted Ping-Pong ball. The painting of the ball has a mechatronics reason. The painting on the very regular ball causes desired irregularities. A standard PingPong ball is precisely defined which cause arbitrary movements in the air flow. The painting modifies these characteristics. Particularly an inhomogeneous surface with a typical paint-drop at one point causes an asymmetric characteristic that can help modifying the stability of the ball. The effect obviously is very sensitive and hard to reproduce but the tendency is pretty much the same with painted balls. The effect that can be observed is that the center of gravity is properly defined in the direction of the paint-drop. When the ball is levitated the paint-drop is oriented downwards. Due to the air flow the ball has a tendency to rotate and to move lateral that is normally not intended. With the painted ball the rotation axis can be set to vertical. With additional air flow guidance within the metal body the ball can be influenced to move almost only vertically with almost no rotation.

\section{System Design}

As mentioned previously, the main objective was to evaluate the use of OCE with the test bench. The used system architecture is an industrial motion-controller hosting the motion-control firmware with motion-kernel and PLC-kernel connected to different execution environments. The motion-controller itself has a local bus interface that can be used to connect different kind of industrial peripherals as there are digital and analog inputs and outputs or communication devices. For the test set-up analog input and output channels were used to connect the Hoverball test bench. The analog output provides the set-point voltage for the fan speed and an analog input that is connected with the ultrasonic position sensor that measures the ball position within the acryl pipe. The motion-controller supports several additional fieldbus technologies like PROFIBUS DP, PROFINET, Ethernet/IP or sercos automation bus. During the evaluation the local bus interface was used only because of the additional time-delays that come with the different bus technologies. Communication dead-times destabilize the control system. Therefore it should be considered during modeling and control parameter settings but it was not in the center of interest during this project.

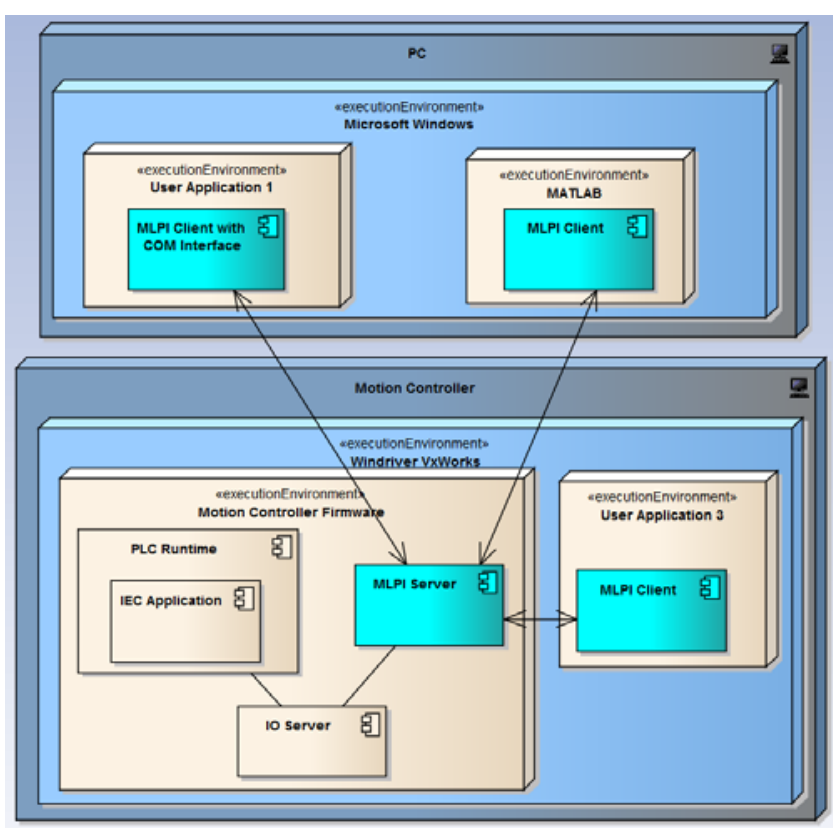

Figure 3: System architecture based on the Open Core Engineering

Fig. 3 shows a general overview of the system architecture in terms of an UML deployment diagram. The fundamental system architecture and some more details are described in $[6,7]$. In the context of this project the key component is the execution environment Firmware at the lower left side. The classic approach of a motion-controller is the execution of a PLC program written in IEC 61131-3 languages as part of the software. This software is executed as real-time component. For the evaluation of the OCE with the Hoverball test bench explained above, the execution environment Windows $P C$ was used but also substituted by or put in parallel to another execution environment Android smartphone. The Ethernet connection can be materialized by a local area network (LAN) or a wireless network (WLAN) and the execution environments can access the motion-controller in parallel.

First of all the test bench was put into operation with a standard IEC 61131-3 software program to determine a controller structure and to find reasonable control parameters as this is the reliable real-time environment. In parallel to this classic design approach a model of the Hoverball was created with MATLAB/Simulink. The system parameters were identified and converted to model parameters to simulate the ball behavior. With this model the parameters and structure of the control algorithm were optimized to get an acceptable system characteristic for the objectives of this project. Fig. 4 shows the measurement of the ball position as reaction to commanded sinusoidal set-points. The signal has a frequency of $80 \mathrm{mHz}$ and a magnitude of $50 \mathrm{~mm}$ at an offset of $500 \mathrm{~mm}$. The ball position follows the commanded set-points with a phase-shift and an intended offset nicely. This means the process can be observed easily and modifications of the system of control parameters are very transparent. As control algorithm an adaptive PID-controller was used. As the controller was not in the center of interest for this paper it will not be 
explained here. However, it should be mentioned that the Hoverball does not have constant model parameters for all ball positions. The system can be separated in three areas. If the ball lies at the bottom of the acryl pipe or if it reaches the end of the pipe, the system changes significantly. This is because aerodynamics changes a lot at the air inlet and outlet. Within the area of $100 \mathrm{~mm}$ to $700 \mathrm{~mm}$ the system characteristics remain quite constant.

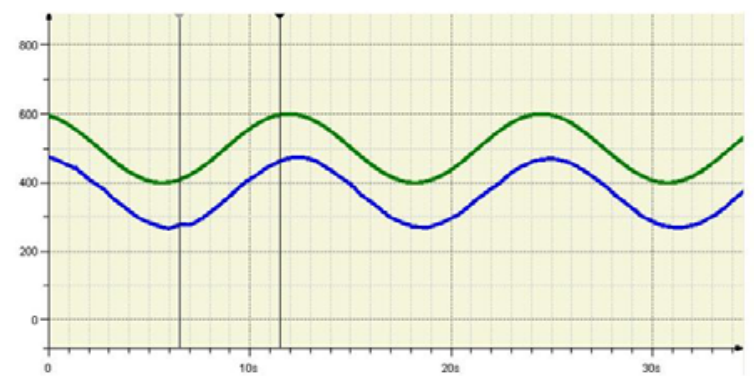

Figure 4: Reaction of the ball position (lower curve) to a commanded sinusoidal set-point (upper curve) in millimeters versus time on the horizontal axis [1]

In order to validate some of the functionality of OCE the control algorithm designed with Simulink was replicated with a MATLAB $m$-file and executed in a Windows PC execution environment (compare Fig. 3). A detailed description of the MATLAB programming interface for OCE was presented in [8]. The results that are not depicted here showed a similar result as shown in Fig. 4. However, as expected with a non-real-time (NRT) environment communicating via a LAN, the cycle time is not constant and the control algorithms work fine only if the system load on the NRT PC does not delay the execution of the control algorithm for more than $50 \mathrm{~ms}$. As a conclusion it is interesting that the control algorithm can stabilize the ball position if system load and communication traffic are respected. For demonstration and evaluation purposes this is acceptable for an industrial application it is not. However, the use of the NRT environment for hard-real-time applications is not the objective. For this purpose real-time environments with Open Core technology are available.

In order to evaluate the test bench with OCE running on a tablet PC or smartphone, an Android based smartphone was selected and a human machine interface for set-point inputs and actual value visualization was implemented in Java. As with the MATLAB control algorithms this implementation is a NRT environment with the additional challenge that communication uses a WLAN link that is shared with other communication nodes and therefore is non-deterministic and cannot be used for control algorithms needing almost stable cycle times of less than $50 \mathrm{~ms}$. This is why the set-point input and process visualization was implemented only. The Java app interfaces the real-time target running the IEC 61131-3 control algorithm and commands the set-points according to the user inputs. Fig. 5 shows the screen to move the ball position with finger swipes.

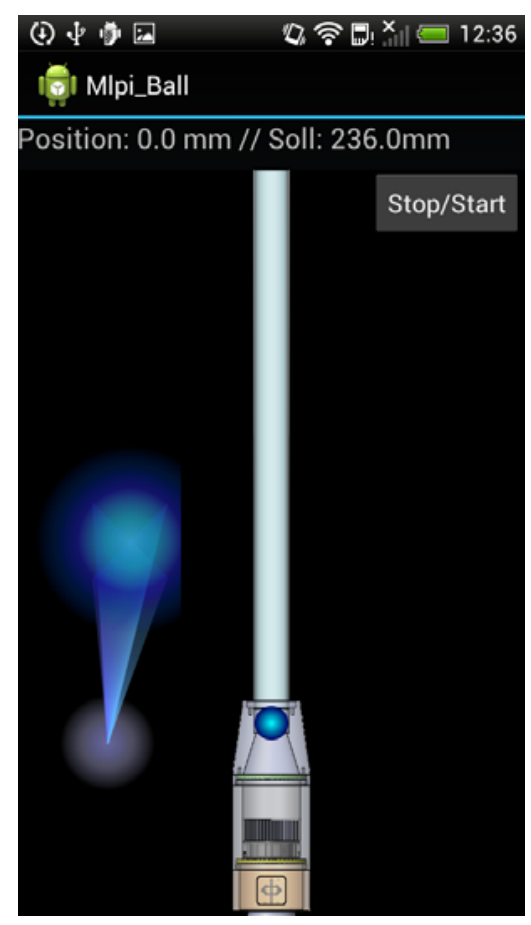

Figure 5: GUI to control the Hoverball with a smartphone [1]

\section{Pre-study forlmprovements}

Following the positive results of the hard- and software tests the design and the construction of the Hoverball was reviewed regarding cost. The initial design was started with a target cost lower than $1000 €$ for the mechanical parts. The target was reached but it is pretty much for a prototype. Within the context of rapid-prototyping technologies a pre-study was conducted to evaluate the manufacturability with fused-layer-modeling (FLM) technology. Fused-layer-modeling is one of the low-cost $3 \mathrm{D}$ printing technologies. It basically describes an additive manufacturing technology based on 3dimensional extrusion of thermoplastics like ABS, Nylon or PLA [10]. As the process is additive it allows production of workpieces that are slightly more complicated than classically produced workpieces. On the one hand side this gives more freedom for design aspects but on the other hand the technology comes with additional constraints, particularly for overhanging construction elements. Another constraint is robustness of the printed material.

Fig. 6 shows as a good example the Hoverball's lower part according to the original construction for production from aluminum and the redesign for manufacturing with FLM technology. The aluminum parts were design to be mounted on top of each other and fixed with screws which needs threading holes as shown in the figure. FLM components are made from layered thermoplastic which means that mounting screws parallel to a layer, which is the case here, is not very rigid. Instead of these threading holes so called screw nut boxes were designed. Before assembly screw nuts can be placed in these boxed and after assembly of parts these boxes fix the nuts and screws can be mounted from the exterior. 
Another aspect is a overhanging element. To produce overhanging elements, FLM technology allows designing support material areas that create a support element bearing the overhanging element during production. After production this material can be removed. However, removing support elements usually causes visual marks that had to be avoided here due to aesthetic design requirements. Depending on production parameters overhanging elements can be produced if the angle between element and vertical axis is in the range of $60^{\circ}$ or higher. In this design overhanging elements were reduced adding slopes with more than $60^{\circ}$.

The initial construction designed for aluminum material has a separate fan grid at the bottom side as it is easier and more rigid to mount a stainless steel grid than to mill the contour with curvatures in aluminum. This could be done in FLM as well but integrating the grid directly in the lower part is possible as well. It reduces the number of parts and assembly effort as well.
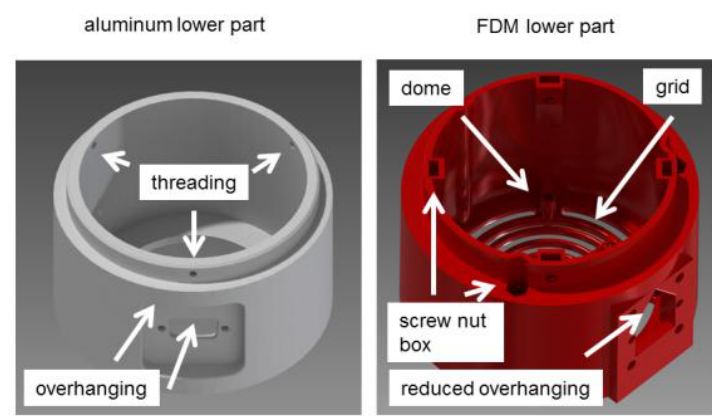

Figure 6: Lower part of the Hoverball in aluminum and lower part in FLM

This was targeted for the mounting of the PCBAs as well. Milling complex structured with small dimensions is hard to be produced with standard milling tools and machines. But FLM gives more flexibility and the workpiece can be more complicated. Therefore the mounting domes for the PCBAs were integrated similar to the fan grid at the bottom of the part. Integral keys were added as well to improve the rigidity of the domes.

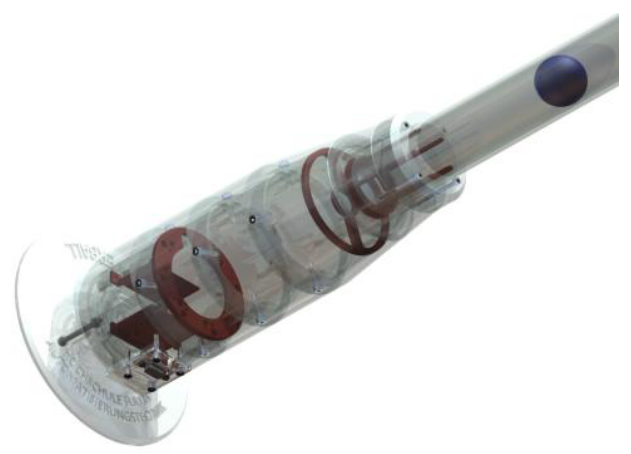

Figure 7: Transparent view of the FLM construction

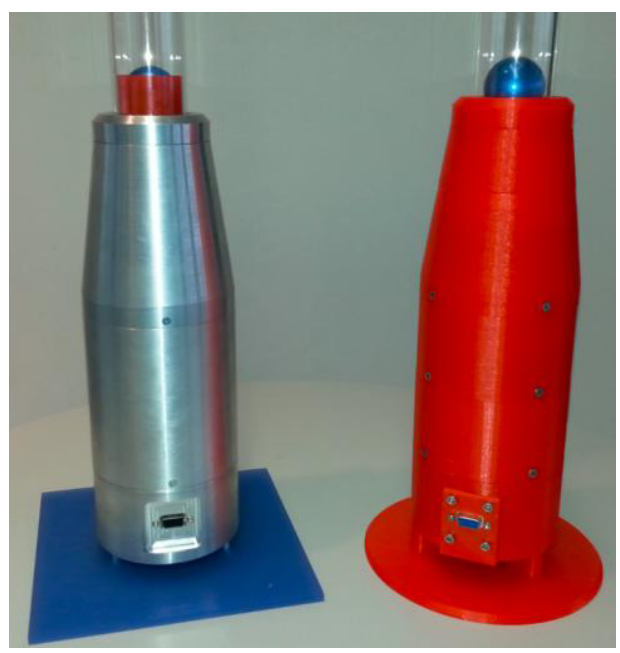

Figure 8: Final aluminum and FLM Hoverball

The lower part of the Hoverball is the most sophisticated FLM part. Other parts were constructed and produced similarly. Fig. 7 shows the complete construction of the FLM Hoverball in a transparent view. The aerodynamics of both constructions is similar but not exactly identical. On the one hand side this is mainly caused be the difference in the fan grids and on the other hand side by the slightly different surface structure of the milled aluminum compared to the rougher surface of FLM components which were not grinded after production.

The major objective designing the FLM Hoverball was the evaluation of the feasibility of producing it with FLM technology and secondly the reduction of production cost. As mentioned above, the aluminum mechanics cost several hundreds of Euros and it took about 4 weeks lead time to get parts. The FLM Hoverball was produced with about $2 \mathrm{~kg}$ of PLA, which is depending on material supplier - less than $100 €$ but the printing time of the components was about 80 hours.

\section{Summary and Conclusion}

The aerodynamic ball levitation test bench, the Hoverball, presented in this paper is the result of several research activities in the context of RCP in industrial automation partially described in $[6,7,8,9]$ extended by research results of a $3 \mathrm{D}$ printable version. It builds a control system that has system characteristics adapted to the performance of industrial motion-controllers not needing additional control electronics. With its tuned complexity and dynamics it is well designed for evaluation purpose but combines at the same time multi-physics like aerodynamics, ultrasonic measuring and a low voltage electric drive. The paper describes the construction and functionality of the Hoverball. It is used in three use cases evaluating the applicability of OCE for this test bench in non-real-time and real-time environments. The use cases cover the software development with IEC 61131-3 programming languages, MATLAB/Simulink modeling and a Java app for an Android smartphone. 
Particularly the implementation of a stabilizing control algorithm running on a non-real-time execution environment like Microsoft Windows has shown that it is possible to use it but it is at the limit and requires dedicated conditions.

Finally the paper describes how the construction can be modified to turn it from a classical aluminum design into a $3 \mathrm{D}$ printable design. The design aspects for $3 \mathrm{D}$ printing are explained and a final version is shown as prove of the feasibility.

\section{References}

1. A. Kindler, Konstruktion, Inbetriebnahme und Implementierung eines Hoverball Labor-modells mit IndraWorks und Android. Bachelor thesis, UAS Aachen, unpublished (2013)

2. Quanser: Products and Solutions. http://www.quanser.com/Products/, $\quad$ accessed 10.03.2014

3. Festo: Lernsysteme. http://www.festo-didactic.com, accessed 10.03.2014

4. Vernier: PID Ping-Pong Ball Levitation. http://www.vernier.com/files/sample labs/EPV-12pid ping pong ball.pdf, accessed 01.06.2013

5. M. Rawashdeh, B. Bowers, Levitating ball using a fuzzy control system http://markbowers.org/levitating-ball, accessed 01.06 .2013
6. E. Engels, H. Schnabel, Rapid-Control-Prototyping of Industrial Drives for the sercos Automation Bus. Mechatronics (MECATRONICS), $20129^{\text {th }}$ FranceJapan $\& 7^{\text {th }}$ Europe-Asia Congress on and Research and Education in Mechatronics (REM), $201213^{\text {th }}$ Int'l Workshop on, pp.177-181, 21-23 Nov. 2012, IEEEXplore

7. E. Engels, S. Krauskopf, Innovation in Motion-Logic programming - a versatile interface, in: Proceedings to the $12^{\text {th }}$ International Workshop on Research and Education in Mechatronics, REM 2011, Kocaeli, Turkey, 15.09.-16.09.2011, ISBN 978-975-8047-963

8. E. Engels, Industrieantriebe mit sercos Schnittstelle über MATLAB ${ }^{\circledR}$ steuern - Konzeption einer MATLAB $^{\circledR}$ Toolbox für die Industrieautomation, AALE 2013, Stralsund, 28.02.-01.03.2013, in: Proceedings pp. 223-232, ISBN 978-3-8356-3364-3

9. E. Engels, Entwicklung einer aerodynamischen Regelstrecke für die multi-disziplinäre Lehre in der Industrieautomation, AALE 2014, Regensburg, 08.05.-09.05.2014, in: Proceedings pp. 31-39, ISBN 978-3-8356-7142-3

10. A. Gebhardt, Generative Fertigungsverfahren: Additive Manufacturing und 3D Drucken für Prototyping - Tooling - Produktion, Carl Hanser Verlag GmbH \& Co. KG, ISBN 978-3446436510 (2013) 